\section{Rectification Suppression in Magnetic-Ge Heterojunction Diode Shown to Be Magnetization-Dependent}

The potential for spin-polarized electronics to advance solid-state electronics by enabling logic and memory to be integrated into a single device or chip has prompted research efforts focused on spin-dependent effects in semiconductors. While electronic spin devices have previously shown relatively small effects, a team of researchers from the Department of Physics and Astronomy at the University of North Carolina in Chapel Hill has demonstrated the viability of producing multifunctional spin devices.

As reported in the August 4 issue of Applied Physics Letters, F. Tsui, L. Ma, and L. He have observed magnetizationdependent diode behavior in a heterojunction consisting of a CoMn-doped $p$-type Ge magnetic semiconductor grown epitaxially on a lightly doped $n$-type Ge substrate. Under electrical bias, the current rectification of the diode can be suppressed by applying a magnetic field. The researchers grew magnetic germanium (M-Ge) films of the form $\mathrm{Co}_{2 x} \mathrm{Mn}_{x} \mathrm{Ge}_{1-3 x}$ on $\mathrm{Ge}(001)$ substrates using molecular-beam epitaxy (MBE) techniques to create heterojunctions with $x<0.05$ (see figure). However, the researchers only achieved smooth two-dimensional M-Ge growth and created heterojunctions with a good low-temperature, magnetization-dependent rectification effect for doping concentrations of $x<0.03$.

Below the $150^{\circ} \mathrm{C}$ Curie temperature $\left(T_{\mathrm{c}}\right)$ of this M-Ge film, a magnetizationdependent suppression of the current rectification effect, which was shown to be a consequence of the film's ferromagnetic order, was reported. Under reverse bias and zero magnetic field $(\mathbf{B}=0)$, the diode operated in the "on state" whereas, at either high magnetic field or under forward bias, the diode operated in the "off state" causing suppression of electron injection into the Ge substrate. At low temperature and a bias of $-2 \mathrm{~V}$, a current ratio of 30 was observed, which corresponds to a field-dependent current ratio, $\Delta I / I_{0}$, of $97 \%$, that is, the ratio of the difference between the zero-field current and the current with an applied magnetic field, $\Delta I=I_{0}-I(\mathbf{B})$, to the zero-field current, $I_{0}=$ $I(\mathbf{B}=0)$. With a field on the order of 100 Oe, the current rectification was suppressed to half of its zero-field value. According to Tsui, "the low magnetic field required to cause the rapid and large suppression of current rectification makes this Ge-based magnetic heterojunction diode a technologically important device."

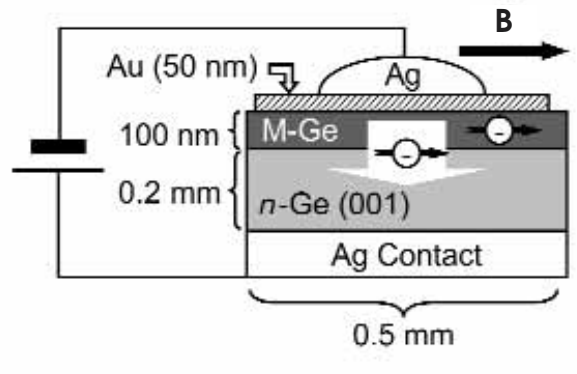

Figure. Schematic of the heterojunction with a doped magnetic $\mathrm{Ge}(\mathrm{M}-\mathrm{Ge})$ film grown epitaxially on an n-type $\mathrm{Ge}(001)$ substrate (n-Ge). Reproduced with permission from Applied Physics Letters 83 (2003); ( 2003 American Institute of Physics.

By examining the field-dependent current ratios as a function of the state variable for magnetization-dependent phenomena, $\mu_{\mathrm{B}} \mathbf{B} / k_{\mathrm{B}} T$ (where $\mu_{\mathrm{B}}$ is the Bohr magneton and $k_{\mathrm{B}}$ is the Boltzmann constant), the researchers demonstrated that the low field current ratios of the device behave differently than those of nonmagnetic Ge depending on the bias directions. These observations differ from previously reported phenomena including magnetoresistance effects in magnetic multilayers or field-dependent carrier trajectory effects, both of which would result in the same field dependence regardless of bias direction. In their initial description of the rectification-suppression phenomena observed in this study, the researchers speculate that there exists a nearly halffilled impurity band in the $p$-type M-Ge film, which is spin-split below $T_{\mathrm{c}}$ such that it is half-metallic in zero field and insulating in high field.

"This magnetization-dependent diode is exciting," said Tsui, "because it not only exhibits a large field-dependent suppression of current rectification at low temperature, but it is also based on a materials system that is structurally and electronically compatible with current Si-based device technology. This work is a result of our intense effort on materials synthesis."

STEFFEN K. KALDOR

\section{Coated Microcantilevers Detect Plastic Explosives}

High explosives such as pentaerythritol tetranitrate (PETN) and hexahydro-1,3,5triazine (RDX) exhibit very low vapor pressures, in the range of parts per trillion at ambient temperatures. Currently, the most sensitive detection techniques report limits on the order of hundreds of picograms, but the technologies involved are often expensive and not easily miniaturized. A team of researchers from the
Oak Ridge National Laboratory and the University of Tennessee has recently employed coated microcantilevers in a detection method that offers limits of detection of a few femtograms and has the potential to be portable and reusable.

As reported in the August 18 issue of Applied Physics Letters, the researchers took advantage of the ability of microcantilevers to sensitively respond to surface-analyte interactions. These interactions induce large surface forces and, if limited to one surface, lead to bending of the cantilever. The team used silicon microcantilevers, which were coated on one side with gold. Each cantilever was immersed in a solution of 4-mercaptobenzoic acid (4-MBA) to produce a uniform, self-assembled monolayer of acid molecules on the gold surface. This carboxyl-terminated monolayer was selected because of its ability to interact with nitro-substituted explosive molecules through hydrogen bonding.

The coated cantilever was held in a vacuum-tight flow cell, and a vapor stream with a trace concentration of PETN or RDX was passed over it. The light of a laser diode was reflected by the gold surface of the cantilever and used to determine both bending response and resonance frequency. Response of the cantilevers to both explosive compounds was clear and fast, with maximum bending observed in 20-25 s. After the stream containing explosive vapors was turned off, the cantilever quickly relaxed to its starting position and could be used for further detections. For both PETN and RDX, the limit of detection was determined to be on the order of several femtograms.

These results demonstrate direct vapor detection of plastic explosives at partsper-trillion levels. Because of the small size of the cantilevers as well as the speed and reversibility of this detection method, the team said that this work "can lead to the development of a portable detection device for rapid and sensitive detection of explosive vapors."

CATHERINe Oertel

\section{Ga-Filled Single-Crystalline MgO Nanotube Serves as Thermometer with a Wide Temperature Range}

Tubular metal oxide nanostructures combine the multifunctionality of carbon nanotubes and the industrially important applications of oxides. However, established template methods for fabricating oxide nanotubes produce polycrystalline structures, which form weaker nanostructures than do single-crystalline nanotubes. In addition, while carbon nanotubes filled with liquid metals have been previously studied, the encapsulation of liquid metals 